\title{
Nóta ar Aicme Éagothroimí idir Shuimeálaithe
}

\author{
J. N. FLAVIN
}

Tiomnaithe dóibh siúd, sa Ghaillimh agus lasmuigh di, a thug an Ghaeilge chun foirfeachta mar mheán pléite eolaíochta agus matamaitice. $* * * * * * * * * * * * * * * * * * * * * * *$

"All analysts spend half their time hunting through the literature for inequalities which they want to use and cannot prove."

curtha i leith Harald Bohr ag G.H. Hardy (1928)

\begin{abstract}
This note concerns some interesting inequalities (essentially) involving integrals with non-negative integrands which depend on a function (of one variable) and its derivatives. One first proves some identities - based on changes of variable - from which simple versions of the inequalities immediately follow: all this is elementary, well within the reach of anyone with a good first course in Calculus. One obtains stronger versions of the inequalities by applying the Wirtinger inequality, and other similar inequalities, to the identities. The meaning of the term 'sharp' inequality is recalled, and it is pointed out that all the inequalities arising are, in a sense, sharp.
\end{abstract}

\section{RÉAMHRÁ}

Sa nóta seo cuirtear ar fáil trí h-ionannais idir shuimeálaithe, bunaithe ar mharlartaithe athróga, ón ar féidir éagothroimí simplí a ghiniúint. Bheadh an chuid seo den nóta (i.Ranna §2-4) ar chumas micléinn (mhaith) Ardteistiméireachta (Ardleibhéal).

Má chuirtear éagothroime Wirtinger (e.g. [1]) agus cinn ar aon dul leis i bhfeidhm ar na h-ionannais réamhluaite gintear éagothroimí níos láidre (§5). Sé an cur chuige seo an ghné is tábhachtaí den nóta: cur chuige éifeachtach é chun teacht ar aicme úsáideach éagothroimí idir shuimeálaithe nach bhfuil fáil ortha go héasca sa litríocht. Ní hí is aidhm don nóta seo, áfach, liosta cuimsitheach a sholáthar de na h-éagothroimí gur féidir d'fháil ar an gcaoi seo. 
Tugtar chun chuimhne sa nóta seo an chiall atá le h-éagothroime ghéar. Is den tsaghas seo na cinn uile i $\S 5$.

Tríd is tríd, is éagothroimí idir shuimeálaithe le h-insuimeálaithe neamhdhiúltacha, a bhraitheann ar fheidhm (aon-athróga) agus a díorthaíocha, na cinn is ábhar don nóta seo. Éagothroimí den aicme ginearálta seo, is minic a úsáidtear iad chun meastacháin cháilíochtúla d'fháil ar réiteacha cothromóidí páirtdhifreálacha faoi choinníollacha ginearálta. Mar shampla, éagothroimí den tsaghas áirithe a bpléitear anseo, úsaidtear iad i [2] chun meastacháin d'fháil don ráta spásúil lena meathlaíonn an réimse struis i bpláta dronuilleogach cuarlíneach leaisteach, faoi choinníollacha áirithe. Toisc nach rabhthas in ann teacht ar an aicme éagothroimí iarluaite i gceann de na lámhleabhair is cuimsithí ar an ábhar [1] is ea spreagadh an cur chuige is ábhar don nóta seo. Ní miste a rá, áfach, go bhfuil an aicme éagothroimí anseo gaolmhar le h-éagothroime a luaitear le Hardy [e.g. [3]] agus le cinn a bpléitear i [4], mar shampla.

\section{Nodaireacht agus Comhthéacs}

Ciallaíodh $r, t$ dhá athróg neamhspleácha ceangailte de réir

$$
r=e^{t} ;
$$

sainmhínítear $r$ san eatramh $a \leq r \leq b$ in ar tairisigh dheimhneacha iad $a, b$, agus, dá réir, sainmhínítear $t$ i $A \leq t \leq B$ in a mbíonn, ar son na h-áisiúlachta,

$$
A=\log a, B=\log b,
$$

agus (rud a úsáidfear ar ball)

$$
L=\log (b / a) .
$$

Ciallaíodh $\psi(r), f(t)$ dhá athróg spleácha ceangailte de réir

$$
\psi=r^{p} f
$$

in ar tairiseach ar bith é $p$.

Feidhmeanna leanúnacha iad $\psi, f$, agus pé díorthaíocha a ndéanfar tagairt dóibh tríd síos, glactar leis go mbíonn siad leanúnach (ach i gcás amháin nuair a chuirtear a mhalairt in iúl). Cuirtear difreáil i leith $r$ agus $t$ in iúl le ' agus ' faoi seach agus difreáil faoi dhó le "agus • faoi seach. Úsáidtear, leis, an nodaireacht atá coitianta i gcomhthéacs suimeála: sainmhínítear an earr-dhifríocht

$$
g(r)]_{a}^{b}=g(b)-g(a) \quad \text { etc. }
$$




\section{NA hIONANNAIS}

Is féidir na h-ionannais seo leanas a chruthú gan ró-dhua (rud a fhágtar faoin léitheoir):

(a)

(b)

$$
\int_{a}^{b} r^{-(2 p+1)} \psi^{2} d r=\int_{A}^{B} f^{2} d t
$$

$$
\left.\int_{a}^{b}\left[r^{-(2 p-1)} \psi^{\prime 2}-p^{2} r^{-(2 p+1)} \psi^{2}\right] d r=\int_{A}^{B} \dot{f}^{2} d t+p f^{2}\right]_{A}^{B}
$$

(c)

$$
\begin{gathered}
\int_{a}^{b}\left[r^{-(2 p-3)} \psi^{\prime \prime 2}-\left\{p^{2}+(p-1)^{2}\right\} r^{-(2 p-1)} \psi^{\prime 2}+p^{4} r^{-(2 p+1)} \psi^{2}\right] d r \\
\left.-r^{-(2 p-1)} \psi^{\prime}\left\{(2 p-1) r \psi^{\prime}-2 p^{2} \psi\right\}\right]_{a}^{b}=\int_{A}^{B} \ddot{f}^{2} d t
\end{gathered}
$$

Tugtar faoi ndeara gur féidir an earr-dhifríocht (feic §2) i (6) agus (7) araon a chur i dtéarmaí $f(t)$ nó $\psi(r)$ : cé acu foirm is áisiúla le n-úsáid amach anseo, braitheann sé ar an gcomhthéacs.

Tharlódh gurbh fhéidir tuilleadh ionannas ar aon dul le (5), (6), (7)a chruthú ina mbíonn díorthaíocha d'ord níos aoirde; nó, gurbh fhéidir, fiú, leagan ginearálta de na h-ionannais a chruthú.

\section{4. ÉAgothroimí Simplí}

Gineann (6) éagothroime shimplí, láithreach, ag cur (4) san áireamh:

\section{Tairiscint 1.}

$$
\int_{a}^{b} r^{-(2 p-1)} \psi^{\prime 2} d r \geq p^{2} \int_{a}^{b} r^{-(2 p+1)} \psi^{2} d r
$$

má's feidhm ar bith i $\psi$ a shásuíonn ceann amháin de na coinniollacha seo leanas:

(i)

(ii)

$$
a^{-p} \psi(a)= \pm b^{-p} \psi(b)
$$

$$
\psi(a)=0 \text { ach } p>0
$$

$$
\psi(b)=0 \text { ach } p<0
$$


Séard a thuigtear le h-éagothroime ghéar sa chomhthéacs ginearálta seo ná ceann a mbíonn cothroime inti d'fheidhm áirithe nach ionann í agus an fheidhm nialasach. Dá réir sin, is léir ó (6), (4), gur éagothroime ghéar í (8) sa bhfochás (i), ach an sín + a thógaint i (9): bíonn cothroime ann i gcás na feidhme

$$
r^{-p} \psi=\text { tairiseach }(\neq 0)
$$

(agus ansin amháin). Fág is nach éagothroime ghéar í (8) - mar a míníodh thuas — sna fochásanna (ii), (iii), is féidir cothroime de shaghas áirithe a bhaint amach in (8) sna fochásanna úd nuair a dhruideann $b / a \rightarrow \infty$ (ar a dtugtar cothroime asamtóiteach): má sainmhínítear $\psi$ de réir (1), (2), (4) agus

$$
f=M \sin \left\{\pi(t-A)(B-A)^{-1}\right\},
$$

in ar tairiseach treallach é $M$, is féidir a chruthú, ag úsáid (5), (6), go ndruideann

$$
\int_{a}^{b} r^{-(2 p-1)} \psi^{\prime 2} d r /\left\{p^{2} \int_{a}^{b} r^{-(2 p+1)} \psi^{2} d r\right\} \rightarrow 1
$$

nuair a dhruideann $b / a \rightarrow \infty($ ach $p \neq 0)$. Dob fhéidir a rá dá réir : ní féidir uimhir níos mó ná $p^{2}$ a chur ina ionad i (8), a bhraitheann ar $p$ amháin (i.e. nach mbraitheann ar $a, b$, leis).

Gineann (7) éagothroime shimplí eile, láithreach:

\section{Tairiscint 2.}

$\int_{a}^{b}\left[r^{-(2 p-3)} \psi^{\prime \prime 2}+p^{4} r^{-(2 p+1)} \psi^{2}\right] d r \geq\left\{p^{2}+(p-1)^{2}\right\} \int_{a}^{b} r^{-(2 p-1)} \psi^{\prime 2} d r$

má's feidhm ar bith i $\psi$ a shásuíonn ceann amháin de na coinníollacha seo leanas:

(i)

$$
a^{-p} \psi(a)= \pm b^{-p} \psi(b) ; a^{-p+1} \psi^{\prime}(a)= \pm b^{-p+1} \psi^{\prime}(b),
$$

(ina ngabhann an dá + agus an dá - le chéile)

(ii)

$$
\begin{aligned}
& \psi^{\prime}(a)=0, \psi(b)=0, \text { ach } 2 p-1>0, \\
& \psi^{\prime}(b)=0, \psi(a)=0, \text { ach } 2 p-1<0,
\end{aligned}
$$

$$
\psi^{\prime}(a)=\psi^{\prime}(b)=0
$$


Is léir gur éagothroime ghéar í (14) sa bhfochás (i), ach an sín + a thógaint i (15): de bharr (7), (4), baintear cothroime amach i gcás na feidhme tugtha ag (12), agus ansin amháin. Dar ndóigh, bíonn an éagothroime (14) bailí nuair a bhíonn an earr-dhifríocht (feic §2) i (7) neamh-dheimhneach: luaitear na coinníollacha (15)-(18) go sonrach chun na críche sin toisc gurb iad is simplí agus is nádúrtha, dar leis an údar. Bíonn na h-éagothroimí (8), (14) bailí, leis, nuair nach mbíonn $a$ deimhneach, ach coinníollacha áirithe a bheith sásuithe, e.g. $2 p+1<0$.

\section{5. ÉAGOThroimí NÍOS LÁIDRE}

Tugtar éagothroime Wirtinger agus a macasamhla, a bhaineann leis an bhfeidhm $f(t)$, san Aguisín: luaitear trí chás - (a), (b), (c). Cuirtear iad i bhfeidhm ar na h-ionnanais (6), (7) chun leaganacha níos láidre a ghiniúint de na h-éagothroimí simplí i §4. Neartaítear (8) trí (a), (c) a chur i bhfeidhm ar (6), ag tosnú leis an gcéad cheann: Ag úsáid (a), (6), (5), gheibhtear

Teoirim 1. Bionn

$$
\int_{a}^{b} r^{-(2 p-1)} \psi^{\prime 2} d r \geq\left(p^{2}+\delta L^{-2}\right) \int_{a}^{b} r^{-(2 p+1)} \psi^{2} d r
$$

má's feidhm ar bith i $\psi$ a shásuíonn (10) nó (11) nó

$$
\psi(a)=\psi(b)=0,
$$

ina chiallaíonn $\delta$ tairiseach deimhneach a mbionn na luacha seo leanas aige sna fochásanna éagsúla:

fochásanna (10), (11): sé $\lambda=\delta$ an préamh (deimhneach) is lú den chothromóid

$$
\lambda^{1 / 2} \cos \lambda^{1 / 2}+|p| L \sin \lambda^{1 / 2}=0
$$

fochás (20):

$$
\delta=\pi^{2} \text {. }
$$

Bíonn cothroime $i$ (19) ins na fochásanna éagsúla, mar leanas: fochásanna (10), (20): nuair a bhíonn

$$
\psi=M r^{p} \sin \left[\delta^{1 / 2} \log (r / a) L^{-1}\right],
$$

in ar tairiseach treallach é $M$, agus ansin amháin;

fochás (11): nuair a bhíonn

$$
\psi=M r^{p} \sin \left[\delta^{1 / 2} \log (b / r) L^{-1}\right],
$$

in ar tairiseach treallach é $M$, agus ansin amháin. 
Tugtar anois leagan eile níos láidre de (8). Ag baint úsáide as (1), (2), (4), (6) agus (c) (Aguisín), gheibhtear

Teoirim 2. Biodh $\rho, p$ de réir $a<\rho \leq b$ agus $p>0$. Má's feidhm ar bith $i \quad \psi(r)$ a shásuíonn $\psi(a)=0$, sásuítear an éagothroime ghéar

$$
\int_{a}^{b} r^{-(2 p-1)} \psi^{\prime 2} d r \geq p^{2} \int_{a}^{b} r^{-(2 p+1)} \psi^{2} d r+m \rho^{-2 p} \psi^{2}(\rho)
$$

ina mbíonn

$$
m=(1+p L)\{\log (\rho / a)\}^{-1}\{1+p \log (b / \rho)\}^{-1} .
$$

Fágtar faoin léitheoir a chruthú gur éagothroime ghéar í seo (feic(c), Aguisín); agus a leithéid d'éagothroime d'fháil sa bhfochás (11), agus sa chás ina mbíonn $\psi(a)=\psi(b)=0$. Tugtar faoi ndeara gur féidir an téarma deiridh ar an dtaobh dheis de (23) a chur i riocht suimeála, má úsáidtear feidhm Dirac: feidhm "ghinearálaithe" is ea feidhm Dirac $\delta(r-\rho)$, a shasuíonn

$$
\int_{a}^{b} \phi(r) \delta(r-\rho) d r=\phi(\rho),
$$

ina mbíonn $a<\rho<b$ agus in ar feidhm leanúnach í $\phi(r)$; feic [5], m.sh.

Ar an gcuma céanna mórán, is féidir (14) a neartú. De ghrá na simplíochta, cloítear leis an gcás ina sásuíonn $\psi$ na coinníollacha (neartaithe)

$$
\psi(a)=\psi^{\prime}(a)=\psi(b)=\psi^{\prime}(b)=0 .
$$

Cuirtear Aguisín (b) i bhfeidhm ar (7), ag nótáil (6) agus

$$
f(A)=\dot{f}(A)=f(B)=\dot{f}(B)=0,
$$

agus gheibhtear

Teoirim 3. Má's feidhm ar bith i $\psi$ a shásuíonn (25), bionn

$$
\begin{aligned}
& \int_{a}^{b}\left[r^{-(2 p-3)} \psi^{\prime \prime 2}+p^{2}\left(p^{2}+4 \pi^{2} L^{-2}\right) r^{-(2 p+1)} \psi^{2}\right] d r \\
& \quad \geq\left\{p^{2}+(p-1)^{2}+4 \pi^{2} L^{-2}\right\} \int_{a}^{b} r^{-(2 p-1)} \psi^{\prime 2} d r
\end{aligned}
$$

bínn cothroime ann sa chás seo leanas (agus ansin amháin):

$$
\psi=M r^{p}[1-\cos \{2 \pi \log (r / a) / L\}]
$$

in ar tairiseach treallach é $M$. 
Míníodh i $\$ 4$ conus is féidir "cothroime asamtóiteach" a bhaint amach i (8) i gcásanna áirithe, nuair $b / a \rightarrow \infty$. Ar an gcuma céanna mórán, is léir ó Theoirim 3 go mbaintear "cothroime asamtóiteach" amach i (14) i gcás na feidhme (27): nuair a dhruideann $b / a \rightarrow \infty$, druideann

$$
\frac{\int_{a}^{b}\left[r^{-(2 p-3)} \psi^{\prime \prime 2}-(p-1)^{2} r^{-(2 p-1)} \psi^{\prime 2}\right] d r}{p^{2} \int_{a}^{b}\left[r^{-(2 p-1)} \psi^{\prime 2}-p^{2} r^{-(2 p+1)} \psi^{2}\right] d r} \rightarrow 1,
$$

ach $p \neq 0$.

Is féidir an éagothroime (14) a neartú ar an-chuid bhealaí eile, nuair a shásuíonn $\psi$ na coinníollacha neartaithe (25) nó nuair a shásuíonn sí ceann amháin de na coinníollacha (16)-(18). Bíonn siad seo go léir bunaithe ar éagothroimí a shásuíonn $f(t)$.

\section{Aguisín: ÉAgothroime Wirtinger agus a Macasamhla}

Is tairisigh iad $B, A, p$ sa chaoi go mbíonn $B>A, p \geq 0$ tríd síos.

(a) Má's feidhm ar bith í $f(t)$

(i) a shásuíonn

$$
f(A)=f(B)=0,
$$

bíonn

$$
\int_{A}^{B} \dot{f}^{2} d t \geq \pi^{2}(B-A)^{-2} \int_{A}^{B} f^{2} d t
$$

(ar a dtugtar Éagothroime Wirtinger);

(ii) a shásuíonn $f(A)=0$, bíonn

$$
\int_{A}^{B} \dot{f}^{2} d t+p f^{2}(B) \geq \delta(B-A)^{-2} \int_{A}^{B} f^{2} d t
$$

ina chiallaíonn $\lambda=\delta$ an préamh (deimhneach) is lú de

$$
\lambda^{1 / 2} \cos \lambda^{1 / 2}+p(B-A) \sin \lambda^{1 / 2}=0 .
$$

Bíonn cothroime ann san éagothroime dheiridh nuair a bhíonn

$$
f=M \sin \left\{\delta^{1 / 2}(t-A)(B-A)^{-1}\right\},
$$

in ar tairiseach treallach é $M$ (agus ansin amháin). Bíonn cúrsaí amhlaidh i (i), leis, ach $\delta=\pi^{2}$.

(b) Má's feidhm ar bith í $f(t)$ a shásuíonn

$$
f(A)=\dot{f}(A)=f(B)=\dot{f}(B)=0
$$


bíonn

$$
\int_{A}^{B} \ddot{f}^{2} d t \geq 4 \pi^{2}(B-A)^{-2} \int_{A}^{B} \dot{f}^{2} d t
$$

bíonn cothroime ann sa chás

$$
f=M\left[1-\cos \left\{2 \pi(t-A)(B-A)^{-1}\right\}\right]
$$

in ar tairiseach treallach é $M$, agus ansin amháin.

Pléitear na h-éagothroimí (a), (b) thuas i [1].

(c) Bíodh $\eta$ de réir $A<\eta \leq B$. Feidhm ar bith $f(t)$ a shásuíonn $f(A)=0$, sásuíonn sí an éagothroime ghéar

$$
\int_{A}^{B} \dot{f}^{2} d t+p f^{2}(B) \geq r(\eta-A)^{-1} f^{2}(\eta)
$$

ina mbíonn

$$
r=\{1+p(B-A)\}\{1+p(B-\eta)\}^{-1} .
$$

Tugtar cruthúnas simplí ar seo toisc nach bhfuil a leithéid ar fáil go héasca sa litríocht, go bhfios don údar.

Cruthúnas: Cuirtear éagothroime Schwarz i bhfeidhm ar an dá ionannas follasacha

$$
f(\eta)=\int_{A}^{\eta} \dot{f} d t, f(\eta)-f(B)=-\int_{\eta}^{B} \dot{f} d t
$$

agus gheibhtear

$$
\begin{gathered}
f^{2}(\eta) \leq(\eta-A) \int_{A}^{\eta} \dot{f}^{2} d t \\
\{f(\eta)-f(B)\}^{2} \leq(B-\eta) \int_{\eta}^{B} \dot{f}^{2} d t .
\end{gathered}
$$

Uatha sin, gheibhtear

$$
\begin{gathered}
(\eta-A)(B-\eta)\left\{\int_{A}^{B} \dot{f}^{2} d t+p f^{2}(B)\right\} \geq(B-A) f^{2}(\eta)+ \\
(\eta-A)\left[\{1+p(B-\eta)\} f^{2}(B)-2 f(\eta) f(B)\right] .
\end{gathered}
$$

Cuirtear an cruthúnas i gcrích trín chearnóg a chomhlánú sa téarma deiridh.

Fágtar faoin léitheoir a chruthú gur éagothroime ghéar an ceann seo (nod: baintear cothroime amach i gcás feidhme a mbíonn díorthaíoch neamhleanúnach aici ag pointe amháin.) 
Focal Buíochais. Táim fíorbhuíoch den Dr. A. Christofides agus den Dr. J. McDermott a chuir comhairle orm maidir le cur i láthair an ailt. Mise amháin atá freagrach as cruinneas na matamaitice. Buíochas ó chroi, leis, le Noelle Gannon, rúnaí na roinne, a phróiseáil an lámhscríbhinn le slacht.

\section{TAGAIRTÍ}

[1] D. S. Mitrinovic, J. E. Pecaric and A.M. Fink, Inequalities involving functions and their integrals and derivatives, Mathematics and its Applications 53, Kluwer Academic Publishers, Dordrecht, Boston, London 1991.

[2] J. N. Flavin and B. Gleeson, On Saint Venant's principle for a curvilinear rectangle in linear elastostatics, J. Math. Mech. Solids 8 (2003), $337-348$.

[3] A. Kufner and L. E. Persson, Weighted inequalities of Hardy type, World Scientific 2003.

[4] W. J. Coles, Wirtinger-type integral inequalities, Pacific J. Math. 11 (1960), 871-877.

[5] I. Richards and H. Youn, Theory of distributions: a non-technical introduction. Cambridge University Press 1990. 
ar bith: any

asamtóiteach: asymptotic

athróg: variable

bailí: valid

braitheann: depends

cearnóg a chomhlánú: to

complete square

comhthéacs: context

cothroime: equality

cothromóidí páirtdhifreálacha:

part. diff. equations

cruthúnas: proof

cur chuige: approach

deimhneach(a): positive

difreáil: differentiation

díorthaíoch(a): derivative(s)

druideann: tends

éagothroime(í): inequality(ies)

earr-dhifríocht: end-difference

eatramh: interval

feidhm(eanna): function(s)

feidhm ghinearálaithe: generalised

function

feidhm nialasach: zero function

fochás: subcase

$\mathrm{g}(\mathrm{h})$ éar: sharp

giniúint: to generate

inshuimeálaithe: integrands

ionanna(i)s: identity(ies)

leanúnach: continuous

J.N. Flavin,

Roinn na Fisice Matamaiticiúla,

Ollscoil na hÉireann,

Gaillimh,

james.flavin@nuigalway.ie macasamhla: (its) like

meastacháin cháilíochtúla:

qualitative estimates

malartaithe: changes

meathlaíonn:decays

neamh-dheimhneach:non-positive neamh-dhiúltach: non-negative

neamhleanúnach: discontinuous

neartú: strengthen

neamhspleách(a): independent

níos láidre: stronger

nodaireacht:notation

ord:order

pláta dronuilleogach

cuarlíneach leaisteach: curvilinear

rectangular elastic plate

préamh: root

réimse struis: stress field

réiteach $(\mathrm{a})$ : solution( $\mathrm{s})$

sainmhínítear: define (passive)

spásúil: spatial

spleách(a): dependent

suimeálaí: integral

shuimeálaithe: integrals

tairisigh dheimhneacha:

positive constants

tairiseach: constant

treallach: arbitrary

Received on 23 January 2004 and in revised form on 16 November 2004. 